\title{
A Case Control Study of Diabetes During Pregnancy and Low Milk Supply
}

\author{
Sarah W. Riddle ${ }^{1}$ and Laurie A. Nommsen-Rivers ${ }^{2}$
}

\begin{abstract}
Objective: The objective of this study was to determine whether a history of diabetes during pregnancy, as a marker of perinatal glucose intolerance, increases the odds of a diagnosis of low milk supply at a Breastfeeding Medicine Clinic (BMC).

Materials and Methods: A case-control analysis was conducted of electronic medical records for BMC visits $<90$ days postpartum. Diabetes was defined as documentation of gestational, type 1, or type 2 diabetes. Cases were defined as those with a low milk supply diagnosis but without latch or nipple problems, and controls as those with latch or nipple problems but without low milk supply. A sensitivity analysis was then conducted by expanding cases to include all low milk supply diagnoses, and controls to include any diagnoses except low milk supply. Odds ratios (OR) and 95\% confidence intervals (CI) for diabetes were calculated in cases versus controls, including adjustment for cesarean delivery, preterm birth, polycystic ovary syndrome, hypothyroidism, and infertility.

Results: In the primary analysis, $14.9 \%$ of 175 cases versus $6.2 \%$ of 226 controls had a history of diabetes during pregnancy (OR 2.6 [95\% CI 1.3-5.2]; adjusted OR 2.4 [95\% CI 1.2-4.9]). In the sensitivity analysis, $14.9 \%$ of 249 cases versus $6.1 \%$ of 312 controls had diabetes in pregnancy (adjusted OR 2.4 [95\% CI 1.4-4.3]). Conclusions: Women diagnosed with low milk supply were significantly more likely to have had diabetes in pregnancy compared with women with latch or nipple problems and, more generally, compared with women with any other lactation difficulty. Further research is needed to elucidate how maternal glucose intolerance may impede lactation.
\end{abstract}

\section{Introduction}

$\mathbf{H}^{2}$ UMAN MILK IS THE OPTIMAL food for human infants and provides known benefits to both mother and infant. ${ }^{1}$ There are significant risks associated with formula use, including increased risk for respiratory and gastrointestinal infection, sudden infant death syndrome, obesity, types 1 and 2 diabetes, and necrotizing enterocolitis. ${ }^{1}$ The majority of mothers in the United States initiate breastfeeding. However, only about half of these mothers reach their breastfeeding goals, and only $19 \%{ }^{2}$ reach the goal of 6 months of exclusive breastfeeding set by multiple professional organizations. ${ }^{3,4}$

Low milk supply is a frequently cited reason why mothers stop breastfeeding earlier than planned. ${ }^{5,6}$ Other than advising mothers to increase the frequency and thoroughness of breast emptying, there are no evidence-based strategies for helping mothers to increase milk supply. ${ }^{7}$ Greater insights into the physiologic causes of low milk supply could lead to new treatment strategies. Emerging evidence suggests that correlates of glucose intolerance such as obesity are associated with delayed lactogenesis (delayed onset of copious milk production in the early postpartum), ${ }^{8,9}$ but it is not known if glucose intolerance is associated with difficulty in sustaining milk production.

This study sought to determine the association between glucose intolerance and diagnosed low milk supply using a case-control study design. It was hypothesized that mothers diagnosed with low milk supply (cases) would have significantly greater odds of diabetes during pregnancy (either gestational diabetes or pre-existing type 1 or 2 diabetes) compared with mothers with a diagnosis of nipple or latch problems (controls). If the hypothesis were confirmed, it would lend support to the concept that interventions targeting insulin action may be a promising and novel strategy toward improving milk supply in vulnerable mothers.

\section{Materials and Methods}

\section{Study design and source population}

A case-control study design was enacted using existing electronic medical records. The source population was

Divisions of ${ }^{1}$ General and Community Pediatrics and ${ }^{2}$ Neonatology, Cincinnati Children's Hospital Medical Center, Cincinnati, Ohio. 
comprised of mothers $\leq 90$ days postpartum presenting for a first encounter to the Breastfeeding Medicine Clinic (BMC) at Cincinnati Children's Hospital Medical Center over a 2-year period (June 1, 2011-May 31, 2013). Thus, the source population is comprised of mothers from the greater Cincinnati region with a strong enough commitment to breastfeeding to seek professional help to overcome any barriers to continued lactation. The study time period represents encounters occurring prior to the physicians at the Center for Breastfeeding Medicine having an awareness of glucose intolerance being a possible risk factor for low milk supply.

The clinic is a referral-based practice of physician lactation consultants who are all board certified in pediatrics and lactation (MD, IBCLC), in a large tertiary care children's hospital. Mothers and infants are both registered as patients and evaluated at the same clinic visit. A standard medical history and a birth history are reviewed with each mother at the first visit, including a history of type 1 or type 2 diabetes or a history of gestational diabetes with this pregnancy. A breast exam and feeding evaluation, including pre- and post-feeding infant weights, are also completed at each visit.

\section{Case definition}

The intention was to include mothers who were experiencing insufficient breastmilk production, despite their infant feeding well at the breast. Therefore, cases were defined as mothers with an encounter diagnosis of "suppressed lactation" but without a diagnosis indicative of an infant latch or maternal nipple problem (as the latter could be caused by poor infant latch). This diagnosis would only be assigned after history, physical, and feeding evaluation (typically including pre- and post-feeding weights) determined that mother was not making enough milk for the infant to breastfeed exclusively. Mothers presenting with perceived low milk supply who were not found to have milk supply problems during the visit would not be given this diagnosis.

\section{Control definition}

The control group was defined as women with encounter diagnoses indicative of a latch or nipple problem but without a "suppressed lactation" diagnosis. Diagnosis codes in the control group included: "poor latch on, postpartum"; "nipple pain"; " mastodynia", " "cracked nipple"; or "infection of nipple." Thus, controls included mothers who had sufficient breastmilk production, despite experiencing discomfort or difficulty with their infant feeding at the breast. Despite breastfeeding challenges, these mothers presumably were able to have sufficient breast emptying to maintain milk production through feeding at the breast and/or pumping.

\section{Primary exposure of interest}

The primary objective was to determine if impaired glucose tolerance during pregnancy — as indicated by a diagnosis of gestational, type 1 , or type 2 diabetes - increased the risk of low milk supply after delivery. Documentation of type 1 , type 2 , or gestational diabetes was used as an indicator of past and/or present glucose intolerance. The medical history, pregnancy history, flow sheet sections, and encounter diagnoses of all records were comprehensively searched within the source population for any notation of diabetes. The medical history section of the electronic medical record includes both type 1 and type 2 diabetes with "yes" or "no" options. Gestational diabetes is available on a list of fixed options under pregnancy complications. Information was extracted through electronic data reports designed by an applications specialist.

\section{Potentially confounding and mediating variables}

The values for additional variables that are relevant to the research question were extracted and routinely recorded into fixed fields during the first visit encounter. All values are based on maternal report at the time of the clinic visit. Potentially confounding variables were: infant and maternal age, and maternal history of polycystic ovary syndrome (PCOS), ${ }^{10}$ hypothyroidism, ${ }^{11}$ or infertility. ${ }^{12}$ In addition, delivery mode and infant prematurity ( $<37$ weeks) were extracted, since both are potential mediators of an indirect relation between gestational diabetes exposure and low milk supply via increased risk of less optimal breast emptying. Maternal body mass index was not available because maternal weight and height are not routinely recorded.

\section{Data analysis}

Logistic regression analysis was used to examine the exposure odds ratio (OR) and $95 \%$ confidence interval (CI) for the association between diabetes exposure and low milk supply. Models were then constructed to examine the odds of low milk supply in those who reported being diagnosed with diabetes during pregnancy (versus no diabetes), after adjusting for confounding and mediating variables. Potentially confounding and mediating variables were included in adjusted models when the $p$ value for its association with either low milk supply or diabetes was $<0.20$ ( $t$ test or chi-square analysis).

A sensitivity analysis was also conducted to determine if the logistic regression results would remain robust to less restrictive definitions of cases and controls. For the sensitivity analysis, the definition of cases was expanded to any mother presenting at $\leq 90$ days postpartum with a diagnosis of suppressed lactation, irrespective of additional diagnoses, including latch or nipple problem. The definition of controls was likewise expanded to any mother presenting at $\leq 90$ days postpartum without a diagnosis of suppressed lactation, irrespective of other diagnoses.

\section{Results}

\section{Study sample}

There were 641 women who had first-encounter visits to the BMC during the designated time period. Of these, 80 women were $>90$ days postpartum and thus excluded from all analyses. Seventy-four of the 561 women who presented at $\leq 90$ days postpartum had both suppressed lactation and a latch problem/nipple diagnosis. Eighty-six women had neither suppressed lactation nor latch problem/nipple diagnosis. Thus, for the primary analysis, controls included 226 women diagnosed with a latch or nipple problem; and cases included 175 women who were diagnosed with suppressed lactation but without nipple symptoms/latch problem (Fig. 1).

In total, 40 records had at least one notation of diabetes in pregnancy. This included 34 women identified with gestational diabetes, four with type 1 diabetes, and two with type 2 diabetes. 


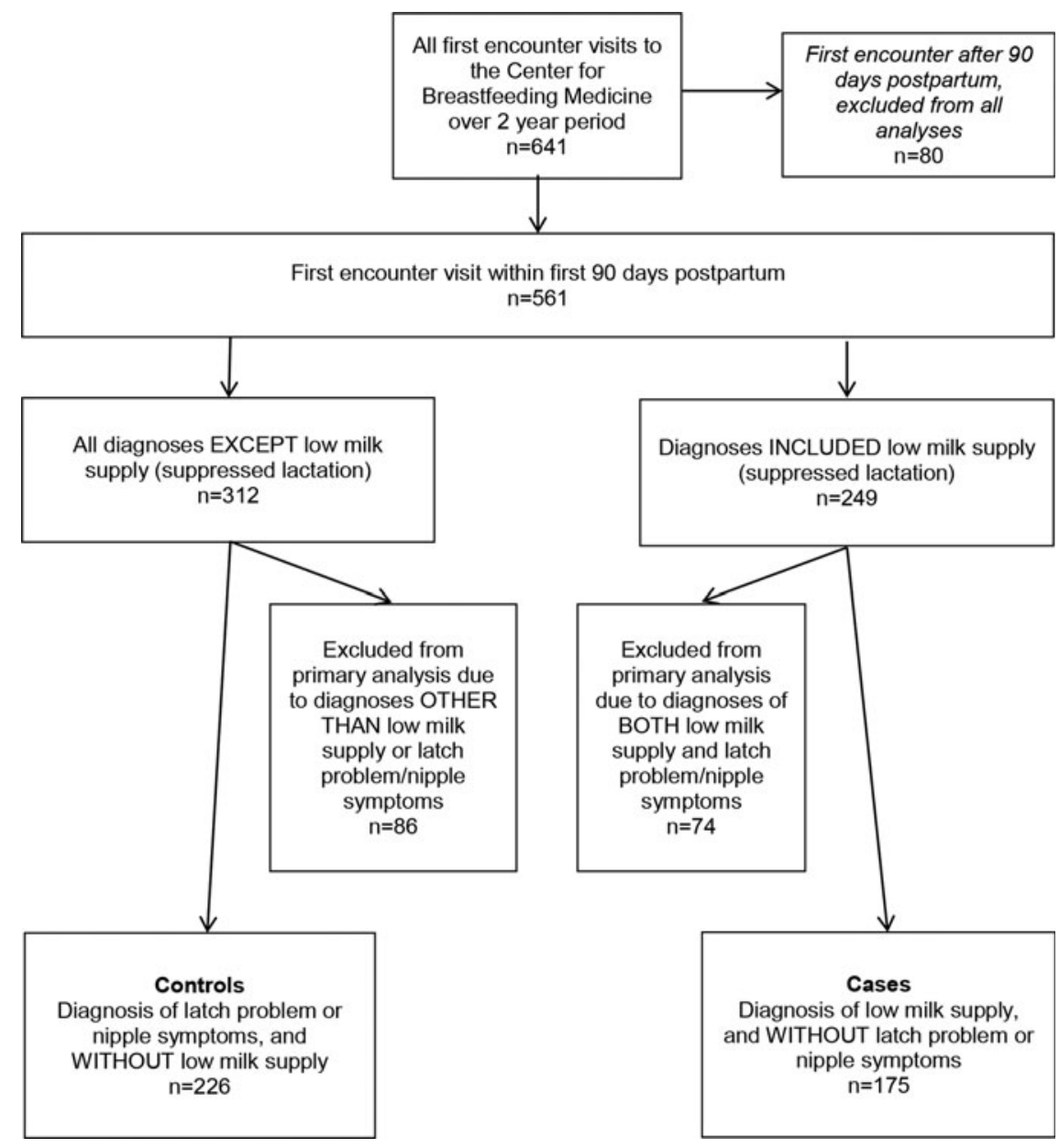

FIG. 1. Derivation of cases and controls from the source population of lactating mothers presenting for a first encounter to the Breastfeeding Medicine Clinic at Cincinnati Children's Hospital Medical Center over a 2-year period (June 1, 2011-May 31, 2013).
Gestational age at delivery was missing for four controls and two cases. Otherwise, potential confounder and mediator data were complete for the 401 women in the analytic data set. For models including gestational age, the sample size was 395 rather than 401 .

Table 1 summarizes the characteristics of cases and controls. Cases were significantly more likely to have had diabetes during pregnancy $(p=0.004)$, a cesarean delivery $(p=0.01)$, or a history of infertility $(p=0.04)$, and displayed a trend toward greater prevalence of preterm delivery $(p=0.10)$ and $\operatorname{PCOS}(p=0.13)$. Cases were not more likely to have a history of hypothyroidism, and were similar in postpartum day of visit and maternal age.

Table 2 presents the odds of low milk supply according to maternal report of diabetes diagnosis and other risk factors. Diabetes in pregnancy was present in $14.9 \%$ of cases $(26 / 175)$

Table 1. Characteristics of Cases and Controls

\begin{tabular}{lcccc}
\hline Characteristic & $\begin{array}{c}\text { Cases } \\
(\mathrm{n}=175)\end{array}$ & $\begin{array}{c}\text { Controls } \\
(\mathrm{n}=226)\end{array}$ & $\begin{array}{c}\text { Cases versus } \\
\text { controls, } \mathrm{p} \text {-value }\end{array}$ & $\begin{array}{c}\text { Diabetes versus } \\
\text { no diabetes } \\
\text { (data not shown), } \mathrm{p} \text {-value }\end{array}$ \\
\hline Diabetes in pregnancy (\%) & 14.9 & 6.2 & $0.004^{\mathrm{a}}$ & - \\
Cesarean delivery (\%) & 37.1 & 25.2 & $0.01^{\mathrm{a}}$ & 0.03 \\
Preterm delivery (\%) & 11.6 & 6.8 & $0.10^{\mathrm{a}}$ & 0.41 \\
Polycystic ovary syndrome (\%) & 8.0 & 4.4 & $0.13^{\mathrm{a}}$ & $0.13^{\mathrm{c}}$ \\
History of hypothyroidism (\%) & 5.1 & 5.3 & $0.94^{\mathrm{a}}$ & $0.29^{\mathrm{c}}$ \\
History of infertility (\%) & 11.4 & 5.8 & $0.04^{\mathrm{a}}$ & $0.06^{\mathrm{c}}$ \\
Postpartum day of visit encounter & $25.1 \pm 18.9$ & $23.2 \pm 17.1$ & $0.29^{\mathrm{d}}$ & 0.43 \\
(mean $\pm S D$ ) & & & $0.8^{\mathrm{d}}$ & 0.41 \\
Maternal age, years (mean $\pm S D)$ & $30 \pm 5.6$ & $30 \pm 4.8$ & & \\
\hline
\end{tabular}

${ }^{\mathrm{a}} p$-Value from chi-square statistic.

${ }^{\mathrm{b}}$ Two cases and four controls missing preterm status.

c $p$-Value from Fisher's exact test.

d $p$-Value from Student's $t$ test.

$S D$, standard deviation. 
Table 2. Odds of Low Milk Supply Diagnosis According to Diabetes in Pregnancy

\begin{tabular}{|c|c|c|}
\hline Model & $\begin{array}{c}A O R \\
{[95 \% C I]^{\mathrm{a}}}\end{array}$ & p-Value \\
\hline $\begin{array}{l}\text { 1. Diabetes in pregnancy } \\
\text { Diabetes, Yes versus No }\end{array}$ & $2.6[1.3-5.2]$ & 0.005 \\
\hline $\begin{array}{l}\text { 2. Diabetes + mediators } \\
\text { Diabetes, Yes versus No } \\
\text { Preterm delivery, Yes versus No } \\
\text { Cesarean delivery, Yes versus No }\end{array}$ & $\begin{array}{l}\mathbf{2 . 5}[\mathbf{1 . 3}-\mathbf{5 . 1}] \\
1.8[0.9-3.7] \\
1.6[1.04-2.5]\end{array}$ & $\begin{array}{l}\mathbf{0 . 0 0 8} \\
0.11 \\
0.03\end{array}$ \\
\hline \multicolumn{3}{|l|}{$\begin{array}{l}\text { 3. Diabetes + mediators + } \\
\text { potential confounders }\end{array}$} \\
\hline Diabetes, Yes versus No & $2.4[1.2-4.9]$ & 0.01 \\
\hline Preterm delivery, Yes versus No & $1.8[0.9-3.7]$ & 0.11 \\
\hline Cesarean delivery, Yes versus No & $1.6[1.02-2.5]$ & 0.04 \\
\hline $\begin{array}{l}\text { Polycystic ovary syndrome, } \\
\text { Yes versus No }\end{array}$ & $1.4[0.5-3.6]$ & 0.51 \\
\hline Infertility, Yes versus No & $1.6[0.7-3.7]$ & 0.26 \\
\hline
\end{tabular}

Statistically significant values are shown in bold.

${ }^{a}$ Logistic regression, OR $[95 \% \mathrm{CI}]$, adjusted for variables specified by model.

AOR, adjusted odds ratio; $\mathrm{CI}$, confidence interval.

and $6.2 \%$ of controls (14/226), resulting in a statistically significant unadjusted OR of 2.6 [95\% CI 1.3-5.2]. The odds of being a low milk supply case if diagnosed with diabetes remained significant after potential mediator adjustment (AOR 2.5 [95\% CI 1.3-5.1]), and after adjusting for both potential mediators and confounding variables (AOR 2.4 [95\% CI 1.2-4.9]). Cesarean delivery was an additional significant risk factor for low milk supply. No other covariate or comorbidity was significantly associated with low milk supply in a fully adjusted model.

A sensitivity analysis was conducted to determine if less restrictive case and control definitions would affect the estimated OR. The definition of cases was expanded to include any mother presenting at $\leq 90$ days postpartum with a diagnosis of suppressed lactation, irrespective of additional diagnoses $(n=249)$, and controls to include any mother presenting at $\leq 90$ days postpartum without a diagnosis of suppressed lactation, irrespective of other diagnoses $(n=312$; Fig. 1). The prevalence of diabetes in cases $(14.9 \%)$ and controls $(6.1 \%)$ using the expanded definitions were nearly identical to the more restrictive definitions used in the primary analysis. Consequently, the sensitivity analysis produced estimated ORs robust to restrictiveness of case and control definitions. In the expanded analysis, the odds of low milk supply in those exposed versus not exposed to diabetes were 2.7 [95\% CI 1.5-4.7], $p=0.0005$ (unadjusted), and 2.4 [95\% CI 1.4-4.3], $p=0.002$ (adjusted as in Table 2, model 3).

\section{Discussion}

Given previously published reports of delayed onset of lactogenesis being predicted by correlates of glucose intolerance such as obesity, older maternal age, and large infant weight, ${ }^{8,13}$ the hypothesis for the present study was that presence of diabetes during pregnancy (as an indicator of abnormal glucose tolerance) may increase the risk of persistent low milk supply. The findings support this hypothesis. Among study controls, defined as women diagnosed with a latch or nipple problem in the absence of low milk supply, $6.2 \%$ had diabetes during pregnancy. This prevalence is equal to the $6.2 \%$ prevalence observed in a systematically sampled prenatal cohort from the same city, ${ }^{9}$ and slightly lower than the reported $8 \%$ prevalence of gestational diabetes for the state of Ohio. ${ }^{14}$ In contrast, $15.9 \%$ of women diagnosed with low milk supply in the absence of latch or nipple problems had diabetes during pregnancy (cases), resulting in 2.6-fold greater odds of exposure in cases than controls. This relation persisted independently of cesarean delivery, preterm birth, PCOS, hypothyroidism, or infertility. In contrast to the assumption that cesarean delivery would mediate an indirect relation between diabetes exposure and low milk supply, cesarean delivery behaved as an independent risk factor, increasing the odds of low milk supply by $60 \%$, but with negligible decline in the point estimate for diabetes (Table 2).

While there have been descriptive studies examining the breastfeeding experience of women with diabetes during pregnancy, ${ }^{15,16}$ to the authors' knowledge, this is the first study to examine the presence of diabetes as a risk factor for low milk supply.

Previous research has reported positive effects of lactation on glucose tolerance. ${ }^{17,18}$ For example, it has been reported that the risk of type 2 diabetes declines as lifetime lactation months increase. ${ }^{18,19}$ In addition, cross-sectional studies in gestational diabetic cohorts report significantly better postpartum glucose tolerance in exclusively breastfeeding women compared with partially breastfeeding or formula-feeding women. ${ }^{17,20}$ These findings have been interpreted as breastfeeding improves metabolic health, and consequently women at risk for diabetes are especially urged to breastfeed. ${ }^{21-24}$ In contrast, the present study is the first to test the reverse relation - that is, whether glucose intolerance causes lactation insufficiency in women attempting to breastfeed. The results support the hypothesis that the same aspects of insulin dysregulation that contribute to gestational diabetes (i.e., waning pancreatic insulin secretion in the context of insulin resistance) may also contribute to sustained difficulties with suppressed production of breastmilk.

While insulin was initially not thought to have a direct role in lactation, ${ }^{25}$ a study in rodents demonstrated that the mammary gland transitions from being predominantly sensitive to Insulin-like Growth Factors I and II (IGF) during mammary growth in pregnancy to becoming highly sensitive to insulin specifically during lactation. ${ }^{26}$ Similarly, insulin-sensitive gene expression in the mammary epithelial cell was recently shown to be strongly upregulated during lactation in humans. ${ }^{27}$ Thus, the present findings are aligned with emerging basic science and clinical evidence ${ }^{8,9}$ pointing to healthy insulin dynamics as an important contributor to timely secretory activation and milk production.

There is widespread belief among clinicians that women diagnosed with PCOS are at risk for low milk production. ${ }^{28}$ However, an independent effect of PCOS on the risk of low milk supply was not observed in a model that included diabetes. The results are consistent with those of Vanky et al., who examined whether prenatal metformin treatment improved lactation outcomes in women diagnosed with PCOS. $^{29}$ As reported by Vanky, breastfeeding duration in the placebo group averaged an impressive 8.5 months, and was not significantly different from the (prenatal) metformin group. However, it is notable that only $18 \%$ of the sample, including the placebo group, developed gestational diabetes. ${ }^{30}$ A possible 
interpretation of these combined results is that: (1) PCOS as a risk factor for insufficient lactation may be limited to the subset of women with postpartum glucose intolerance, and (2) metformin administered prenatally may not be an effective intervention for this condition.

\section{Limitations}

The translation of the adjusted odds ratio result to the more clinically relevant relative risk measure is limited by the unknown prevalence of low milk supply in the larger population from which the study controls arose. For the odds ratio to represent relative risk, low milk supply in the source population would need to be $<5 \%$ prevalence. Low milk supply is a highly prevalent concern of new mothers, but the prevalence of true low milk supply remains unknown. Further study is needed utilizing a prospective cohort design to elucidate better the public health impact of these findings. Covariates in the model were limited by the data available in the electronic medical record. Maternal weight and height were not available because they are not routinely recorded at the visit, thus limiting the ability to adjust for any independent contribution of obesity to low milk supply. Obese mothers are at significantly greater risk of cesarean delivery, ${ }^{31}$ and thus obesity may be an unmeasured confounder in the observed association between delivery mode and risk of low milk supply. However, the odds ratio for diabetes was only slightly attenuated after adjustment for delivery mode, suggesting that glucose intolerance is a risk factor for low milk supply independent of obesity, even if delivery mode is representing residual confounding related to maternal obesity.

Case and control definitions were based on the available encounter diagnosis options as chosen by the provider at the time of the visit. While there were no options that would likely compete with "suppressed lactation" for coding low milk supply, there are multiple options for coding problems that may indicate suboptimal infant breastfeeding behavior, necessitating the expansion of the encounter diagnosis list for signifying latch problems. As a result, among the nipple symptom diagnoses, some but not all represent mothers who were having difficulty with the infant feeding at the breast (and other records may represent mothers who were having nipple or breast pain unrelated to how their infant was feeding at the breast). Nonetheless, all of the controls represent mothers without low milk supply, despite encountering a latch or nipple problem or discomfort.

Diabetes in pregnancy often complicates the maternity course, including greater risk of maternal-infant separation after delivery due to infant complications, neonatal intensive care unit stay, or cesarean delivery. It is possible that, among study cases, some of the infants may have had latch difficulties as newborns that contributed to low milk supply but resolved prior to the first encounter at the BMC. However, the sensitivity analysis revealed that mothers with low milk supply (cases) had the same rate of diabetes as the group of mothers who were excluded because they had diagnoses of both low milk supply and latch/nipple problems (Fig. 1). This finding suggests that the presence of diabetes has a physiologic effect on milk production that is independent of diabetes complications that may interfere with feeding at the breast, because no additive effect of latch/nipple problems on risk of diabetes exposure was observed.

\section{Conclusion}

Utilizing data from a source population of women who were highly motivated to breastfeed, significantly greater odds of diabetes history was observed in women with a low milk supply diagnosis compared with women with latch or nipple problems and, more generally, compared with women with any other lactation difficulty. Two decades of research have consistently shown that obesity is a risk factor for poor lactation outcomes. Even though insulin resistance is common among obese women, no published clinical study has prospectively examined how insulin secretion and sensitivity may impede lactation. Future research may reveal an important role for healthy insulin action in supporting milk production, which opens up an entirely novel venue for the development of targeted therapeutic interventions to treat low milk supply.

\section{Acknowledgments}

We would like to acknowledge Alison Perkins, Application Specialist at Cincinnati Children's Hospital Medical Center (CCHMC), for her technical assistance with data extraction. We acknowledge Dr. Maurizio Macaluso (CCHMC), Dr. William Brinkman (CCHMC), Dr. Courtney Brown (CCHMC), Dr. Kathy Auger (CCHMC), Dr. Samir Shah (CCHMC), and Dr. Patrick Brady (CCHMC) for critical review of this manuscript prior to submission; and we acknowledge Donna Wuest (administrative assistant, CCHMC) for assistance with document formatting.

We would also like to acknowledge support of Dr. NommsenRivers by the National Institutes of Health (NIH) Office of Research on Women's Health through grant 5 K12 HD051953. The content is solely the responsibility of the authors and does not necessarily represent the official views of the NIH.

\section{Disclosure Statement}

No competing financial interests exist.

\section{References}

1. Ip S, Chung M, Raman G, et al. Breastfeeding and maternal and infant health outcomes in developed countries. Evid Rep Technol Assess (Full Rep) 2007;1-186.

2. Centers For Disease Control and Prevention. Breastfeeding report card-United States, 2014. www.cdc.gov/breastfeeding/ pdf/2014breastfeedingreportcard.pdf (accessed October 30, 2015).

3. American Academy of Pediatrics Section on Breastfeeding. Breastfeeding and the use of human milk. Pediatrics 2012; 129:e827-841.

4. World Health Organization. The optimal duration of exclusive breastfeeding. Report of the expert consultation. Geneva, Switzerland, 2001.

5. Li R, Fein SB, Chen J, et al. Why mothers stop breastfeeding: mothers' self-reported reasons for stopping during the first year. Pediatrics 2008;122:S69-76.

6. Wagner EA, Chantry CJ, Dewey KG, et al. Breastfeeding concerns at 3 and 7 days postpartum and feeding status at 2 months. Pediatrics 2013;132:e865-875.

7. Academy of Breastfeeding Medicine Protocol Committee. ABM Clinical Protocol \#9: use of galactogogues in initiating or augmenting the rate of maternal milk secretion (First Revision January 2011). Breastfeed Med 2011;6:41-49. 
8. Nommsen-Rivers LA, Chantry CJ, Peerson JM, et al. Delayed onset of lactogenesis among first-time mothers is related to maternal obesity and factors associated with ineffective breastfeeding. Am J Clin Nutr 2010;92:574584.

9. Nommsen-Rivers LA, Dolan LM, Huang B. Timing of stage II lactogenesis is predicted by antenatal metabolic health in a cohort of primiparas. Breastfeed Med 2012;7:43-49.

10. Vanky E, Isaksen $\mathrm{H}$, Moen $\mathrm{MH}$, et al. Breastfeeding in polycystic ovary syndrome. Acta Obstet Gynecol Scand 2008;87:531-535.

11. Speller E, Brodribb W. Breastfeeding and thyroid disease: a literature review. Breastfeed Rev 2012;20:41-47.

12. Cromi A, Serati M, Candeloro I, et al. Assisted reproductive technology and breastfeeding outcomes: a case-control study. Fertil Steril 2015;103:89-94.

13. Nommsen-Rivers LA, Mastergeorge AM, Hansen RL, et al. Doula care, early breastfeeding outcomes, and breastfeeding status at 6 weeks postpartum among low-income primiparae. J Obstet Gynecol Neonatal Nurs 2009;38:157-173.

14. Ohio Department of Health. Ohio pregnancy risk assessment monitoring system, Center for Public Health Statistics and Informatics, 2012. www.odh.ohio.gov/healthstats/pramshs/ prams1.aspx (accessed March 14, 2014).

15. Soltani H, Dickinson FM, Kalk J, et al. Breast feeding practices and views among diabetic women: a retrospective cohort study. Midwifery 2008;24:471-479.

16. Cordero L, Thung S, Landon MB, et al. Breast-feeding initiation in women with pregestational diabetes mellitus. Clin Pediatr (Phila) 2014;53:18-25.

17. Gunderson EP, Hedderson MM, Chiang V, et al. Lactation intensity and postpartum maternal glucose tolerance and insulin resistance in women with recent GDM: the SWIFT cohort. Diabetes Care 2012;35:50-56.

18. Schwarz EB, Brown JS, Creasman JM, et al. Lactation and maternal risk of type 2 diabetes: a population-based study. Am J Med 2010;123:863.e1-6.

19. Villegas R, Gao YT, Yang G, et al. Duration of breast-feeding and the incidence of type 2 diabetes mellitus in the Shanghai Women's Health Study. Diabetologia2008;51:258-266.

20. O'Reilly MW, Avalos G, Dennedy MC, et al. Atlantic DIP: high prevalence of abnormal glucose tolerance post partum is reduced by breast-feeding in women with prior gestational diabetes mellitus. Eur J Endocrinol 2011;165:953959.

21. Institute of Medicine Committee on Obesity Prevention Policies for Young Children. Early Childhood Obesity Prevention
Policies: Goals, Recommendations, and Potential Actions. Washington, DC: Institute of Medicine, 2011.

22. U.S. Department of Health and Human Services. The Surgeon General's Call to Action to Support Breastfeeding. Washington, DC: Office of the Surgeon General, 2011.

23. Gabbe SG, Landon MB, Warren-Boulton E, et al. Promoting health after gestational diabetes: a National Diabetes Education Program call to action. Obstet Gynecol 2012;119:171-176.

24. Indian Health Service, Division of Diabetes Treatment and Prevention. Breastfeeding Support. Albuquerque, NM: Indian Health Service, 2011.

25. Lawrence RA, Lawrence RM. Physiology of lactation. In: Breastfeeding: A Guide for the Medical Profession, 7th ed. Maryland Heights, MO: Elsevier, 2011, p.70.

26. Berlato C, Doppler W. Selective response to insulin versus insulin-like growth factor-I and -II and up-regulation of insulin receptor splice variant B in the differentiated mouse mammary epithelium. Endocrinology 2009;150: 2924-2933.

27. Lemay DG, Ballard OA, Hughes MA, et al. RNA sequencing of the human milk fat layer transcriptome reveals distinct gene expression profiles at three stages of lactation. PLoS One 2013;8:e67531.

28. Marasco L, Marmet C, Shell E. Polycystic ovary syndrome: a connection to insufficient milk supply? J Hum Lact 2000; 16:143-148.

29. Vanky E, Nordskar JJ, Leithe H, et al. Breast size increment during pregnancy and breastfeeding in mothers with polycystic ovary syndrome: a follow-up study of a randomised controlled trial on metformin versus placebo. BJOG 2012;119:1403-1409.

30. Vanky E, Stridsklev S, Heimstad R, et al. Metformin versus placebo from first trimester to delivery in polycystic ovary syndrome: a randomized, controlled multicenter study. $J$ Clin Endocrinol Metab 2010;95:E448-455.

31. Chu SY, Kim SY, Schmid CH, et al. Maternal obesity and risk of cesarean delivery: a meta-analysis. Obes Rev2007; 8:385-394.

Address correspondence to: Sarah W. Riddle, MD Cincinnati Children's Hospital Medical Center 3333 Burnet Avenue, ML 2011 Cincinnati, $\mathrm{OH} 45229$

E-mail: Sarah.Riddle@cchmc.org 